\title{
Metalloprotein Plasticity: Cautionary Tales
}

\author{
Nicholas J. Schnicker \\ Protein Crystallography Facility, Roy J. and Lucille A. Carver College of Medicine, University of Iowa, \\ 51 Newton Road, Iowa City, IA, 52242
}

Metal ions are incorporated into about one-third of all proteins to serve various roles in both structure and biological function. Almost 40\% ( 51,000) of the structures deposited in the Protein Data Bank (PDB) contain a metal ion. ${ }^{1}$ Modeling metal ions and their protein residue ligand conformations can be challenging for many reasons such as resolution, binding site complexity, and the level of prior characterization of metal binding. This has led to a substantial number of structures that contain an incorrectly modeled metal ion or an unusual coordination environment around the metal ion. ${ }^{2-4}$ We have analyzed a few published metalloprotein crystal structures to illustrate the extra information that can often be extracted or modeled in around the metal sites at high-resolution. This analysis could help explain some of the discrepancies that are commonly seen with low and even medium resolution metalloprotein structures such as metalligand distances and geometries.

1. Putignano V., Rosato A., Banci L., Andreini C. (2018) MetalPDB in 2018: a database of metal sites in biological macromolecular structures. Nucleic Acids Res. 46(D1), D459-D464.

2. Harding M.M., Nowicki M.W., Walkinshaw M.D. (2010) Metals in protein structures: a review of their principal features. Crystallography Reviews. 16, 247-302.

3. Zheng H., Chordia M.D., Cooper D.R., Chruszcz M., Muller P., Sheldrick G.M., Minor W. (2013) Validation of metal-binding sites in macromolecular structures with the CheckMyMetal web server. Nature Protocols. 9, 156-170.

4. Rupp B., Wlodawer A., Minor W., Helliwell J.R., Jaskolski M. (2016) Correcting the record of structural publications requires joint effort of the community and journal editors. The FEBS Journal. 283, 4452-4457. 